\title{
DIFFERENTIAL EQUATIONS INVOLVING A PARAMETRIC FUNCTION ${ }^{1}$
}

\author{
R. H. CAMERON
}

1. Introduction. It is the purpose of this paper ${ }^{2}$ to find conditions on the function $f(t, u)$ under which the differential system

$$
\left\{\begin{array}{l}
\frac{d z}{d t}+f[t, y(t)+z(t)]=0 \\
z(0)=0
\end{array}\right.
$$

has a solution $z(t)$ on the unit interval $I$ for almost all choices of the function $y$ in the space $C$. Here $C$ denotes the space of functions which are continuous on the interval $I: 0 \leqq t \leqq 1$ and which vanish at $t=0$; and "almost all" means all except a set of Wiener measure ${ }^{3}$ zero. Under the transformation

$$
z(t)=x(t)-y(t)
$$

the system (1.1) goes into the equivalent nonlinear integral equation

$$
y(t)=x(t)+\int_{0}^{t} f[s, x(s)] d s,
$$

so that we are seeking conditions on $f$ which make (1.3) have a solution $x \in C$ for almost every choice of $y$ in $C$.

The simplest conditions of this type which we have found, and which do not force (1.3) to have a solution for every $y \in C$, are given in the following theorem.

THEOREM 1. Let $f(t, u)$ have continuous first partial derivations $f_{t}$ and $f_{u}$ in the strip $R: 0 \leqq t \leqq 1,-\infty<u<\infty$, and let $f$ satisfy the three order of growth conditions

$$
\begin{aligned}
f(t, u) \operatorname{sgn} u & \geqq-A_{1} \exp \left(B u^{2}\right) & & \text { in } R, \\
f_{u}(t, u)+4 g_{t}(t, u) & \leqq 2 \alpha^{2} u^{2}+A_{2} & & \text { in } R,
\end{aligned}
$$

Presented to the Society April 20, 1957 ; received by the editors February 15, 1957.

1 This research was supported by the United States Air Force, through the office of Scientific Research of the Air Research and Development Command, under contract No. AF 18(603)-30. Reproduction in whole or in part is permitted for any purpose of the United States Government.

${ }^{2}$ The author wishes to thank Mr. James Yeh for carefully checking the manuscript of this paper.

'See, for instance, [4]. 


$$
g(1, u) \geqq-\frac{1}{2} \alpha u^{2} \cot \beta-A_{3} \text { for all real } u,
$$

where

$$
g(t, u)=\int_{0}^{u} f(t, v) d v
$$

in $R$,

and $A_{1}, A_{2}, A_{3}, B, \alpha, \beta$ are positive constants with $\alpha<\beta<\pi$ and $B<1$. Then it follows that corresponding to almost every choice of $y \in C$, the system (1.1) has a solution $z \in C$, (and of course, this is the only solution defined on the interval $I$ ).

The fact that this can apply in cases where classical theorems (i.e., theorems giving conditions under which there are solutions for all choices of $y$ in $C$ ) do not apply is shown by the counter-example

$$
\begin{aligned}
f(t, u) & =\frac{1}{2} u^{1 / 3} \sin \left(u^{4 / 3}\right)+\frac{1}{2} u^{5 / 3} \cos \left(u^{4 / 3}\right) \\
& =\frac{3}{8} \frac{d}{d u}\left[u^{4 / 3} \sin u^{4 / 3}\right]
\end{aligned}
$$

It is easy to see that (1.8) satisfies the conditions of the theorem, and hence that there are solutions of (1.1) for almost all $y$ in $C$ when $f$ is given by (1.8). On the other hand, we shall show in $\$ 3$ that there exists at least one $y$ in $C$ for which (1.1) has no solution. Thus Theorem 1 cannot be contained in any classical theorem.

2. Proof of Theorem 1. Assume that the hypotheses of the theorem are satisfied, choose $\gamma=B^{-1}-1$, and let

$$
\phi(t, u)=(t+\gamma)^{-1 / 2} \exp \left\{u^{2}(t+\gamma)^{-1}\right\} \quad \text { in } R .
$$

Let

$$
G(t, u, \lambda)=g(t, u)+\lambda \phi(t, u), \quad(t, u) \in R, \lambda \geqq 0,
$$

so that

$$
G_{u}(t, u, \lambda)=f(t, u)+2 \lambda(t+\gamma)^{-1} u \phi(t, u), \quad(t, u) \in R, \lambda \geqq 0
$$

and

$$
G_{u, u}+4 G_{t}=f_{u}+4 g_{t}, \quad(t, u) \in R, \lambda \geqq 0 .
$$

From (2.1), (2.0), (1.4) we have

$$
G_{u} \operatorname{sgn} u \geqq A_{1}
$$


when $|u| \geqq \lambda^{-1} B^{-3 / 2} A_{1}$ and $t \in I$ and $\lambda>0$, and since for fixed positive $\lambda, G_{u}$ is bounded for $|u| \leqq \lambda^{-1} B^{-3 / 2} A_{1}, t \in I$, it follows that there is a positive function of $\lambda$ alone, $A(\lambda)$, such that

$$
G_{u}(t, u, \lambda) \operatorname{sgn} u \geqq-A(\lambda), \quad(t, u) \in R, \lambda>0 .
$$

Now (2.5) implies that for fixed positive $\lambda$, the integral equation

$$
y(t)=x(t)+\int_{0}^{t} G_{u}(s, x(s), \lambda) d s
$$

has a solution $x \in C$ for each $y \in C$. For the equation clearly has a solution on some interval to the right of zero, and if this interval does not include $t=1$, it must be open at the right hand end and the solution must become unbounded in the neighborhood of this point. But by (2.5), this would imply that $y$ would vary by unbounded amounts as $x$ did so, contrary to the assumption that $y$ is continuous.

Since for fixed positive $\lambda,(2.6)$ has a solution for each $y$ in $C$, it follows from Theorem 3 of [3], (using Footnote 9), that

$$
\int_{C} \exp \{J(x, \lambda)\} d_{w} x=1, \quad \lambda>0,
$$

where

(2.8) $J(x, \lambda)=\int_{0}^{1} K(s, x(s), \lambda) d s-2 G(1, x(1), \lambda) \quad$ for $x \in C, \lambda \geqq 0$, and

$$
K(t, u, \lambda)=\frac{1}{2} G_{u, u}-G_{u}^{2}+2 G_{t} \quad(t, u) \in R, \lambda \geqq 0 .
$$

But by (2.9), (2.2), (2.3),

$$
\lim _{\lambda \rightarrow 0^{+}} K(t, u, \lambda)=K(t, u, 0)=\frac{1}{2} f_{u}+2 g_{t}-f^{2} \quad \text { in } R,
$$

and for each fixed $x$ in $C$, we have from (2.8), (2.10), (2.1), (2.2), (2.3), by bounded convergence

$$
\begin{aligned}
\lim _{\lambda \rightarrow 0^{+}} J(x, \lambda) & =J(x, 0) \\
& =\int_{0}^{1} K(s, x(s), 0) d s-2 g(1, x(1)) .
\end{aligned}
$$


Moreover, it follows from (2.8), (2.9), (2.3), (2.1), (1.5), (1.6), that

$$
J(x, \lambda) \leqq \frac{1}{2} \int_{0}^{1}\left\{f_{u}(s, x(s))+4 g_{t}(s, x(s))\right\} d s-2 g(1, x(1))
$$

$$
\leqq \log Q(x)+\frac{1}{2} A_{2}+2 A_{3} \quad \text { for } x \in C, \lambda>0
$$

where for $x \in C$,

$$
Q(x)=\exp \left\{\alpha^{2} \int_{0}^{1}[x(s)]^{2} d s+\alpha[x(1)]^{2} \cot \beta\right\} .
$$

Finally, we show that $Q(x)$ is integrable over $C$, by applying the transformation

$$
y(t)=x(t)-\alpha \int_{0}^{t} \cot [\alpha s+\beta-\alpha] x(s) d s, \quad t \in I
$$

to the Wiener integral of unity, using Theorem A of [2]. We obtain (using (2.13))

$$
1=\int_{C} 1 d_{w} y=\exp \left\{-\frac{1}{2} \alpha \int_{0}^{1} \cot [\alpha s+\beta-\alpha] d s\right\} \int_{C} Q(x) d_{w} x,
$$

so that the integrability of $Q$ is established.

Now we take limits in (2.7) as $\lambda \rightarrow 0^{+}$, using (2.11), (2.12) and dominated convergence, and thus establish that (2.7) holds even when $\lambda=0$. Hence it follows from Theorem 3 of [3], (using Footnote 9) and from (2.8), (2.9), (2.1), (2.2), (2.3), that the integral equation (1.3) has solutions $x \in C$ for almost all $y \in C$. But (1.3) is equivalent to (1.1) by the transformation (1.2), and the theorem is proved.

3. A counterexample. We shall now show that when $f(t, u)$ is given by (1.8), there exists a function $y \in C$ such that (1.3) (and hence also (1.1)), has no solution in $C$. We begin by constructing a certain function $x$ which does not belong to $C$ because it becomes infinite as we approach $t=1$.

Let

$$
t_{n}=1-n^{-1 / 3} \text { and } u_{n}=(2 n \pi)^{3 / 4}, \quad n=1,2,3, \cdots,
$$

let $M$ be the set of monotonically increasing functions defined on $[0,1)$ which satisfy

$$
x(0)=0, \quad x\left(t_{n}\right)=u_{n}, \quad n=1,2, \cdots,
$$

and let $M_{c}$ be the subset of $M$ consisting of those elements of $M$ which are continuous on $[0,1)$. Define the functionals $Q_{n}(x)$ by 


$$
Q_{n}(x)=\int_{t_{n}}^{t_{n+1}} f[x(s)] d s, \quad x \in M,
$$

where $f(u) \equiv f(t, u)$ is given by (1.8). We shall show the existence of an element $x$ of $M_{c}$ for which

$$
Q_{n}(x)=u_{n}-u_{n+1}
$$

for sufficiently large $n$; i.e., for which

$$
x\left(t_{n+1}\right)-x\left(t_{n}\right)+Q_{n}(x)=0
$$

for sufficiently large $n$.

To show that there is an element of $M_{c}$ for which (3.4) holds for all sufficiently large $n$, consider a particular interval $\left[t_{n}, t_{n+1}\right]$ and an element $x_{1}$ of $M$ which is constant on $\left[t_{n}, t_{n+1}\right)$, so that

$$
x_{1}(t)=u_{n}, \quad t_{n} \leqq t<t_{n+1} .
$$

Then we have by (3.3), (3.1), (1.8),

$$
Q_{n}\left(x_{1}\right)=f\left(u_{n}\right)\left[t_{n+1}-t_{n}\right]=\frac{1}{2} u_{n}^{5 / 3}\left(t_{n+1}-t_{n}\right)>0>u_{n}-u_{n+1} .
$$

Now it is clear that $M_{c}$ is dense in $M$ in the $L_{1}\left[t_{n}, t_{n+1}\right]$ topology, and also that $Q_{n}(x)$ is continuous in the $L_{1}\left[t_{n}, t_{n+1}\right]$ topology applied to the space $M$, since $u_{n} \leqq x(t) \leqq u_{n+1}$ when $x \in M$ and $t \in\left[t_{n}, t_{n+1}\right]$. Hence there is an element $x_{2} \in M_{c}$ for which $Q_{n}\left(x_{2}\right)$ differs as little as we please from $Q_{n}\left(x_{1}\right)$, and in particular

$$
Q_{n}\left(x_{2}\right)>u_{n}-u_{n+1} .
$$

To obtain an $x$ where the inequality goes the other way, we now set

$$
x_{3}=u_{n}^{\prime} \equiv[(2 n+1) \pi]^{3 / 4}, \quad t_{n}<t<t_{n+1},
$$

so that we have by (3.3), (3.1), (1.8),

$$
\begin{aligned}
Q_{n}\left(x_{3}\right) & =f\left(u_{n}^{\prime}\right)\left[t_{n+1}-t_{n}\right] \\
& =-\frac{1}{2}[(2 n+1) \pi]^{5 / 4}\left[n^{-1 / 3}-(n+1)^{-1 / 3}\right], \\
& \leqq-\frac{1}{6}[2 n \pi]^{5 / 4}[n+1]^{-4 / 3}
\end{aligned}
$$

while

(3.8) $\quad u_{n}-u_{n+1}=(2 n \pi)^{3 / 4}-[2(n+1) \pi]^{3 / 4} \geqq-\frac{3}{4}(2 \pi)^{3 / 4} n^{-1 / 4}$. 
It is clear that there exists a positive integer $N$ such that for $n>N$, the last member of (3.8) is greater than the last member of (3.7), so that we have

$$
Q_{n}\left(x_{3}\right) \leqq u_{n}-u_{n+1} \quad \text { if } n>N .
$$

Hence it follows from the continuity of $Q_{n}$ in the $L_{1}\left[t_{n}, t_{n+1}\right]$ topology and the density of $M_{c}$ in $M$ that there exists an element $x_{4} \in M_{c}$ for which

$$
Q_{n}\left(x_{4}\right) \leqq u_{n}-u_{n+1} \quad \text { if } n>N .
$$

Now if we put

$$
x(t)=\lambda x_{2}(t)+(1-\lambda) x_{4}(t), \quad t \in I,
$$

it follows from (3.6) and (3.9) and the continuity of $Q_{n}(x)$ that there is a value of $\lambda$ on $(0,1)$ for which (3.4) holds, if $n>N$. Thus for $n>N$, it is possible to choose $x(t)$ on each interval $\left[t_{n}, t_{n+1}\right]$ so as to make (3.4) hold, and these choices can be made independently for each $n>N$. Let such choices be made for each interval $\left[t_{n}, t_{n+1}\right], n>N$, and choose $x$ on the previous intervals in any way which makes $x \in M_{c}$. Using this choice of $x$, we now define $y$ on $[0,1)$ by substituting this $x$ in (1.3). It now follows from (3.4) that for $n>N,(3.5)$ holds, and from (3.3), (3.5), (1.3) that $y\left(t_{n}\right)$ is independent of $n$ for $n>N$. Setting $y(1)$ equal to this constant value, we have

$$
y\left(t_{n}\right)=y(1)
$$$$
n>N \text {, }
$$

and $y(t)$ is now defined everywhere on $I$.

To show that $y$ is left continuous at $t=1$, we assume $n>N$ and $t_{n} \leqq t \leqq t_{n+1}$, and write, using (3.10), (1.8), (3.1) and the monotonicity of $x$,

$$
\begin{aligned}
& |y(t)-y(1)|=\left|y(t)-y\left(t_{n}\right)\right| \\
& \quad \leqq x\left(t_{n+1}\right)-x\left(t_{n}\right)+\int_{t_{n}}^{t_{n+1}}|f[x(s)]| d s \\
& \quad \leqq u_{n+1}-u_{n}+\int_{t_{n}}^{t_{n+1}} u_{n+1}^{5 / 3} d s \\
& \quad=[(2 n+1) \pi]^{3 / 4}-(2 n \pi)^{3 / 4}+[2(n+1) \pi]^{5 / 4}\left[n^{-1 / 3}-(n+1)^{-1 / 3}\right] \\
& \quad \rightarrow 0 \quad \text { as } n \rightarrow \infty,
\end{aligned}
$$

and it follows that $y \in C$. Moreover, this choice of $y$ corresponds to no solution $x \in C$ of (1.3), since the solution is the chosen $x$ and is unique on each interval $[0,1-\epsilon]$, and any solution on $[0,1]$ would have to 
agree on $[0,1)$ with this unbounded $x$ that we chose. Thus it is established that if $f$ is given by (1.8), the equation (1.3) does not have a solution for every $y$ in $C$.

4. Conclusion. For simplicity, we did not state Theorem 1 in its most general form, and it is easy to see from the proof of the theorem that we can weaken two of the hypotheses a little.

THEOREM 2. If we weaken conditions (1.4) and (1.5) of Theorem 1 by replacing them by conditions

$$
f(t, u) \operatorname{sgn} u \geqq-A_{1} \exp \left(\frac{u^{2}}{t+\gamma}\right)
$$

in $R$,

$\left(1.5^{\prime}\right) f_{u}(t, u)+4 g_{t}(t, u) \leqq 2 \alpha^{2} u^{2}+A_{2}+\{\max [0, f(t, u) \operatorname{sgn} u]\}^{2}$ in $R$, where $\gamma$ is a positive constant, it follows that the conclusion of Theorem 1 holds.

Whether or not Theorem 2 is really more general than Theorem 1 is still an open question, as the author has not yet found any function which satisfies the hypotheses of Theorem 2 but not those of Theorem 1.

In a previous paper, [1], the author raised the question whether

$$
y(t)=x(t)+\int_{0}^{t}[x(s)]^{2} d s
$$

has solutions for almost every $y$ in $C$, and he pointed out two other questions which are equivalent to this one. These questions are not answered by this paper, since $f(t, u) \equiv u^{2}$ does not satisfy condition (1.6). They have, however, recently been answered in the negative in an unpublished paper by D. A. Woodward.

\section{REFERENCES}

1. R. H. Cameron, Nonlinear Volterra functional equations and linear parabolic differential systems, Journal d'Analyse Mathématique vol. 5 (1957) pp. 136-182.

2. R. H. Cameron and W. T. Martin, Evaluation of various Wiener integrals by use of certain Sturm-Lionville differential equations. Bull. Amer. Math. Soc. vol. 51 (1945) pp. 73-89.

3. R. H. Cameron and J. M. Shapiro, Nonlinear integral equations. Ann. of Math. vol. 62 (1955) pp. 472-497.

4. N. Wiener, Generalized harmonic analysis. Acta Math. vol. 55 (1930) pp. 117258 (esp. pp. 214-234).

University OF MinNESOTA 